\title{
$\nabla$
}

\section{Dermoscopic patterns of melanocytic nevi in children and adolescents: a cross-sectional study*}

\author{
Christiane Donato Piazza ${ }^{1}$ \\ Aline P Marcassi ${ }^{1}$ \\ Maria P Seize ${ }^{1}$
}

\author{
Sergio Yamada ${ }^{1}$ \\ Marina G Maciel $^{1}$ \\ Silmara C P Cestari ${ }^{1}$
}

DOI: http:/ / dx.doi.org/10.1590/abd1806-4841.20175209

\begin{abstract}
BACKGROUND: Childhood is a dynamic period regarding nevogenesis. Dermoscopy is a noninvasive technique, recommended for the evaluation of pigmented cutaneous lesions.

ОвјестіVEs: The purpose of this study was to describe the structures and dermoscopic patterns of melanocytic nevi observed in children and adolescents.

METHODS: Dermoscopy with photographic documentation was used for nevi located on the face, trunk, and extremities of 38 patients aged from one to 16 years examined at the Pediatric Dermatology Outpatient Clinic of the Federal University of São Paulo. REsults: The study included 201 skin lesions that were diagnosed as nevi during clinic examination. Upon evaluation of the global dermoscopic pattern of the lesions, the most frequently observed nevi were reticular (39.0\%), followed by homogeneous $(23.9 \%)$ and globular nevi $(16.4 \%)$. During evaluation of the dermoscopic structures, according to the body site, the pigment network was the most observed in the extremities.

Study limitations: A limitation to be considered is that the inclusion of small or new lesions may hinder the differentiation between dots and globules.

Conclusions: In our study, the most observed pattern was reticular. There was a difference in the predominance of structures dependent on the anatomical location.
\end{abstract}

Keywords: Child; Dermoscopy; Nevus

\section{INTRODUCTION}

Childhood is a dynamic period with regard to nevogenesis. ${ }^{1}$ Many new melanocytic nevi will appear and grow during childhood and adolescence. ${ }^{2}$

Dermoscopy is a noninvasive technique that allows the visualization of subsurface structures by decreasing reflection at the stratum corneum-air interface. ${ }^{3}$ This technique is ideal for the evaluation of pigmented skin lesions in children, as it is painless and provides important information that can assist physicians in formulating appropriate management decisions. ${ }^{1}$ Nevertheless, studies of dermoscopic nevi patterns in children, which are essential for the understanding of nevogenesis, are still scarce in medical literature. ${ }^{3}$

The present study evaluates 201 cutaneous lesions suggestive of melanocytic nevi in patients aged from one to 16 years, with the intention of describing the observed structures and dermoscopic patterns.

\section{METHODS}

The studied population included 38 children, aged from one to 16 years, who were examined at the Pediatric Dermatology Outpatient Clinic of the Federal University of São Paulo (EPM-UNIFESP) in July and August of 2013. Lesions with macular or papular components of any size, with color varying from light brown to black, diagnosed as melanocytic nevus at clinical examination, were included in the study.

Patients characteristics such as sex, age, and color were documented, being classified as white (Fitzpatrick 1,2,3), brown (Fitzpatrick 4,5), or black (Fitzpatrick 6).

Each patient was evaluated according to the number of nevi, and these lesions were measured in millimeters at their larger axis.

Lesions were divided into three groups according to their location, i.e., face, trunk and extremities (arms and legs, excluding palms and soles).

Received on 24.02.2015

Approved by the Advisory Board and accepted for publication on 13.01.2016

* Study conducted at the Pediatric Dermatology Outpatient Clinic of the Universidade Federal de São Paulo (EPM- UNIFESP) - São Paulo (SP), Brazil. Financial Support: None.

Conflict of interest: None.

1 Dermatology Outpatient Clinic of the Universidade Federal de São Paulo (EPM-UNIFESP) - São Paulo (SP), Brazil.

(C2017 by Anais Brasileiros de Dermatologia 
Photographic documentation of dermoscopy was performed with the DermLite DL3 dermatoscope, coupled with a Nikon J1 camera.

Documented lesions were evaluated by two observers, both dermoscopy experts, one of them the professor responsible for dermoscopy group of UNIFESP.

Evaluation of the lesions was based on the presence of structural components, identified as globules, dots, structureless areas, branched streaks and pigment network.

For the evaluation of the dermoscopic structures present in the lesions, a statistical study was made with the Minitab program, version 16.1, and the Chi-squared test was applied (significance level $\mathrm{p}<0.05$ ).

Lesions on the face were also evaluated for the presence of pseudopigment network (this pattern often appears because the darker facial background is perforated by both pigment-free follicles and openings of sweat glands, causing light holes to appear). Even having specific dermoscopic patterns, nevi on the face were included in the study for evaluation of dermoscopic structures found in these lesions, as well as for comparison with observed body lesions.

Trunk and extremities lesions were classified according to their global pattern (predominant pattern). When possible, face lesions were also classified this way.

Lesions located in other anatomical regions, such as scalp, soles, palms, and mucous membranes, were excluded. ${ }^{4-6}$

This study was approved by the Council Board of the Dermatology Department of UNIFESP.

\section{RESULTS}

Of the 38 children evaluated, 19 were male and 19 were female.

Twenty-one children (55.26\%) were brown, nine $(23.68 \%)$ white, and eight $(21.05 \%)$ black.

The ages varied from one to 16 years, with a mean age of $8.4 \pm 3.5$ years.

The number of lesions per patient varied from one to 20, with a median number of 4 .

A total of 201 lesions were observed: 75 (37.3\%) on the trunk, $81(40.3 \%)$ on the extremities and $45(22.4 \%)$ on the face.

Of the 201 lesions, five $(2.5 \%)$ were reported as being present at birth.

The mean diameter of the lesions was $2.3 \pm 1.4 \mathrm{~mm}$, with 2.5 $\pm 1.4 \mathrm{~mm}$ for trunk lesions, $2.3 \pm 1.6 \mathrm{~mm}$ for extremities lesions, and $1.9 \pm 1.0 \mathrm{~mm}$ for facial lesions.
Dermoscopic structures present in lesions, according to the localization, are presented in table 1.

With respect to the dermoscopic structures evaluated, the prevalence of globules was significantly different $(p=0.020)$ among trunk, extremities and face, being higher on trunk lesions (38.7\%).

The presence of structureless areas was significantly different $(p<0.001)$ among anatomic areas, being higher on trunk lesions $(61.3 \%)$.

The presence of branched streaks was significantly different $(p=0,044)$ among anatomic areas, being higher on trunk lesions $(10.7 \%)$.

The presence of pigment network was significantly different $(\mathrm{p}<0.001)$ among anatomic areas, being higher on extremity lesions $(58.0 \%)$.

Of the 45 facial lesions, $40(88.9 \%)$ presented pseudopigment network.

The most prevalent dermoscopic structures observed in the lesions were dots $(72.6 \%)$, followed by structureless areas $(47.8 \%)$, pigment network $(40.8 \%)$ and globules $(28.4 \%)$. The presence of branched streaks was observed in only $5.5 \%$ of the lesions.

On the face, the prevalence of globules and pigment network was inverted, showing a higher prevalence of globules than of pigment network.

Of the 159 lesions that could be classified according to their global pattern, 75 were found on the trunk, 81 on the extremities and three on the face (Table 2). The most prevalent global pattern was reticular, observed in $62(39.0 \%)$ of lesions, including three lesions on the face. The second most frequently observed pattern was homogeneous in 38 (23.9\%) lesions, followed by globular in $26(16.4 \%)$ lesions (Figure 1).

On the face, 40 lesions showed pseudopigment network and two lesions showed dots, as predominant structure. These 42 lesions weren't classified according to the global pattern.

\section{DISCUSSION}

This study describes the observations of 201 nevi in 38 patients aged from one to 16 years. Of the studied nevi, five $(2.5 \%)$ were referred as congenital. The incidence of congenital nevi is, according to the literature, from $1 \%$ to $6 \%{ }^{7}$

Mean number of nevi per patient was 4 . Aguilera et al. studied 180 patients aged from one to 15 years and reported a median of 9 nevi per patient. ${ }^{7}$ The lower median observed in our study might

TABLE 1: Dermoscopic structures present in lesions, according to the body sites

\begin{tabular}{|c|c|c|c|c|c|c|c|c|c|}
\hline \multirow[t]{2}{*}{ Structures Present } & \multicolumn{2}{|c|}{$\begin{array}{l}\text { Trunk } \\
(\mathrm{N}=75)\end{array}$} & \multicolumn{2}{|c|}{$\begin{array}{l}\text { Extremities } \\
(\mathrm{N}=81)\end{array}$} & \multicolumn{2}{|c|}{$\begin{array}{l}\text { Face } \\
(N=45)\end{array}$} & \multicolumn{2}{|c|}{$\begin{array}{l}\text { Total } \\
(\mathrm{N}=201)\end{array}$} & \multirow[t]{2}{*}{ p-value $^{1}$} \\
\hline & $\mathbf{N}$ & $\%$ & $\mathbf{N}$ & $\%$ & $\mathbf{N}$ & $\%$ & $\mathbf{N}$ & $\%$ & \\
\hline Globules & 29 & $(38.7 \%)$ & 21 & $(25.9 \%)$ & 7 & $(15.6 \%)$ & 57 & $(28.4 \%)$ & 0.020 \\
\hline Dots & 57 & $(76.0 \%)$ & 55 & $(67.9 \%)$ & 34 & $(75.6 \%)$ & 146 & $(72.6 \%)$ & 0.464 \\
\hline Structureless areas & 46 & $(61.3 \%)$ & 39 & $(48.2 \%)$ & 11 & $(24.4 \%)$ & 96 & $(47.8 \%)$ & $<0.001$ \\
\hline Branched streaks & 8 & $(10.7 \%)$ & 2 & $(2.5 \%)$ & 1 & $(2.2 \%)$ & 11 & $(5.5 \%)$ & 0.044 \\
\hline Pigment Network & 32 & $(42.7 \%)$ & 47 & $(58.0 \%)$ & 3 & $(6.7 \%)$ & 82 & $(40.8 \%)$ & $<0.001$ \\
\hline Pseudopigment network ${ }^{2}$ & - & & - & & 40 & $(88.9 \%)$ & - & & - \\
\hline
\end{tabular}

${ }^{1}$ Chi-squared Test, ${ }^{2}$ Evaluated only in lesions on the face 
TABLE 2: Global pattern of lesions, according to the body sites

\begin{tabular}{|c|c|c|c|c|c|c|c|c|}
\hline \multirow[t]{2}{*}{ Pattern } & \multicolumn{2}{|c|}{ Trunk } & \multicolumn{2}{|c|}{ Extremities } & \multicolumn{2}{|c|}{ Face } & \multicolumn{2}{|c|}{ Total } \\
\hline & $\mathbf{N}$ & $\%$ & $\mathbf{N}$ & $\%$ & $\mathbf{N}$ & $\%$ & $\mathbf{N}$ & $\%$ \\
\hline Reticular & 20 & $(26.7 \%)$ & 39 & $(48.1 \%)$ & 3 & $(100 \%)$ & 62 & $(39.0 \%)$ \\
\hline Homogeneous & 19 & $(25.3 \%)$ & 19 & $(23.5 \%)$ & 0 & $(0 \%)$ & 38 & $(23.9 \%)$ \\
\hline Globular & 14 & $(18.7 \%)$ & 12 & $(14.8 \%)$ & 0 & $(0 \%)$ & 26 & $(16.4 \%)$ \\
\hline Reticular homogeneous & 7 & $(9.3 \%)$ & 6 & $(7.4 \%)$ & 0 & $(0 \%)$ & 13 & $(8.2 \%)$ \\
\hline Globular homogeneous & 10 & $(13.3 \%)$ & 2 & $(2.5 \%)$ & 0 & $(0 \%)$ & 12 & $(7.5 \%)$ \\
\hline Unspecified & 5 & $(6.7 \%)$ & 2 & $(2.5 \%)$ & 0 & $(0 \%)$ & 7 & $(4.4 \%)$ \\
\hline Reticular globular & 0 & $(0 \%)$ & 1 & $(1.2 \%)$ & 0 & $(0 \%)$ & 1 & $(0.6 \%)$ \\
\hline Total (lesions) & 75 & $(100 \%)$ & 81 & $(100 \%)$ & 3 & $(100 \%)$ & 159 & $(100 \%)$ \\
\hline
\end{tabular}
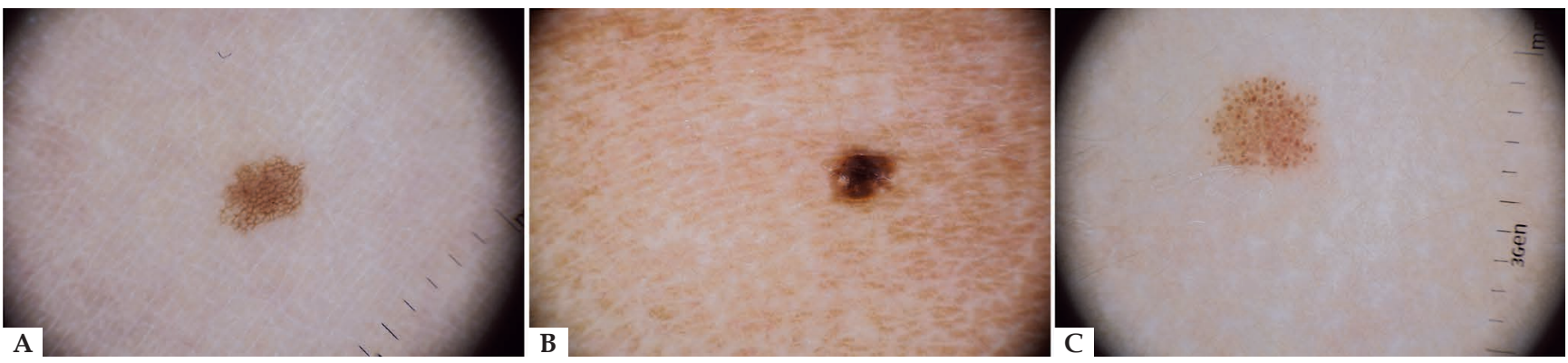

FIGURE 1: Examples of the dermoscopic patterns considered. A. Nevus with a reticular pattern; B. nevus with a homogeneous pattern, C. nevus with a globular pattern. $133 \times 272 \mathrm{~mm}(300 \times 300 \mathrm{DPI})$

result, in part, due to the exclusion of lesions found on the scalp, mucous membranes, soles and palms.

Regarding skin color, it is worth mentioning that studies have reported an association between dermoscopic pattern and the phenotype of the child. Nevi with globular and homogeneous dermoscopic patterns are more frequently observed in fair-skinned children. ${ }^{3}$ Children with darker pigmentation seem to display the reticular pattern more often in their nevi and background skin. ${ }^{8}$ These findings seem related to the higher constitutive activity of melanocytes and/or an enhanced transfer of melanin to keratinocytes. ${ }^{3,9}$ Pigmented keratinocytes highlight the epidermal rete ridge pattern in the background skin as "reticulation", a process that is amplified in nevi. ${ }^{3}$

In our study, as most of the population was brown or black (76.31\%), we also observed a predominance of the reticular pattern (39.0\%). However, a specific evaluation of the dermoscopic pattern in each phenotype was not made. A more detailed documentation of the phenotype (including amount of sun exposure) would allow for a better evaluation of its association with dermoscopic pattern.

The pathogenesis of melanocytic nevi has been debated since the beginnings of histopathology. Likewise, the progeny of nevus cells has been studied; there is some indication that these cells represent melanocytes, differing only in the grade of maturity. ${ }^{9}$

Unna (1896) was most likely the first author to conceptualize the development of nevi. ${ }^{10} \mathrm{He}$ believed that the proliferation of nevus cells at the dermo-epidermal junction would give rise to dermal nevi by the process of Abtropfung (melanocytic nevus cells would develop in the epidermis and "drop off" to the dermis with time). ${ }^{11}$ This action would produce the sequential development of junctional, compound, and finally, dermal melanocytic lesions. ${ }^{9}$ This concept was supported by studies that observed that junctional proliferation of nevus cells is present in almost all melanocytic nevi in children. By contrast, intradermal nevi are uncommon in the first decade of life, and their proportion increases progressively with age.

In 1984, the American pathologist Cramer postulated an intimate cytogenetic relation of melanocytic cells with perineural cells based on immunohistochemical studies. He hypothesized that during ontogenesis, precursor cells, derived from the neural crest, would reach the skin via cutaneous nerves extending from the paraspinal ganglia. Cramer suggested that the concept of Abtropfung should be replaced by the concept of Hochsteigerung (literally: upward climb), meaning an upward melanocyte migration. He interpreted the phenomenon of recurrence of nevi after incomplete surgical excision as proof of this concept. ${ }^{9}$

In the last 20 years, some authors have adhered to the concept of Abtropfung, while others have refuted it, as with the converse concept of Hochsteigerung. Ultimately, there is the possibility that both could represent aspects of nevogenesis. ${ }^{9}$

Nevogenesis has been proposed to occur via two pathways. The "constitutional or endogenous" pathway is thought to give rise to nevi with a globular or structureless dermoscopic pattern (a nonspecific dermoscopic pattern), with onset during childhood. These nevi would derive predominantly from dermal melanocytes and, with time, would appear as intradermal nevi. In contrast, the "ac- 
quired or exogenous" pathway is thought to give rise to nevi with a reticular dermoscopic pattern. The most likely involved exogenous factor is intermittent UV light exposure, resulting in the proliferation of epidermal melanocytes that tend to involute with time. Thus, the pattern of the nevus would be dependent on age. ${ }^{12}$

Reticular nevi can appear and involute during adult life, and globular structureless nevi tend to appear in youth and persist indefinitely. ${ }^{12}$ Some histological studies reported a junctional proliferation that was higher in the first decade, whereas the proportion of dermal nevi showed a constant increase with age from the second decade. However, selection bias must be considered, because the nevi under study represent those excised due to a request by the physician or by the patient. In addition, dermal nevi would, most likely, be detected later, and then excised in an older population than for junctional nevi (with a clearly visible pigmentary component). ${ }^{9}$

The histological exam is a momentary evaluation of a dynamic process but, by definition, the process is permanently halted by biopsy and/or excision. Thus, determining whether a nevus cell population evolves, resides, and regresses, can only occur if the nevus is allowed to "live", i.e., if it is evaluated through noninvasive techniques. ${ }^{9}$ This way, dermoscopy has become a key technique in the evaluation of melanocytic lesions. ${ }^{12}$

According to published studies, nevi with a reticular pattern on dermoscopy correlate with junctional nevi on histopathology. Nevi with a globular pattern on dermoscopy correlate with compound or dermal nevi on histopathology. ${ }^{3}$ However, the globular pattern on dermoscopy (which correlates with compound or dermal nevi, and is expected to appear later) has shown, in other studies, to be predominant in children. ${ }^{1,13-16}$ Therefore, the globular pattern can also be associated with actively growing nevi, and the patterns can change according to age.

Zauladek et al. (14), studying the predominance of dermoscopic structures in acquired melanocytic nevi $(>5 \mathrm{~mm})$ according to age, observed a marked predominance $(82 \%)$ of the globular pattern in the youngest group (0-15 years), thus considering this pattern to be "typical" of childhood nevi. ${ }^{14}$ Oliveria et al. observed a globular pattern in $38 \%$ of the studied cases, suggesting that those nevi would be considered growing or would be congenital. ${ }^{15}$

On the contrary, in our study, the reticular pattern was the most commonly observed (39\%), in agreement with histological studies, which refer to the predominance of junctional nevi in the first decade of life. ${ }^{11}$ According to the body site, the reticular pattern was the most prevalent in the extremities

It is important to remember that the facial lesions we have evaluated (with higher prevalence of globules than pigment network), in their majority, were not classified according to the global pattern because, even when presenting globules, they were not predominant.

The globular pattern is reported to be observed in small congenital melanocytic nevi $(<1.5 \mathrm{~cm}){ }^{13,14}$. In our study, among the five nevi referred to as "congenital", four presented a reticular pattern. Selection bias must be considered, because only nevi present at birth were identified as "congenital".

Variations in melanocytic nevi, dependent on their location on the body, have not been well studied by histological analysis.

There are reports of the predominance in the distribution of dermal nevi ("bulky") mainly on the head and the upper part of the body, whereas junctional nevi are found mainly on distal parts of extremities. It is interesting that, in fetal development, the migration of neural crest cells interferes with basement membrane and hair follicle genesis, leading to a periadnexal distribution of nevus precursor cells. Given that hair follicle growth follows a cephalocaudal sequence, this model can also explain the preference for some nevus types in certain anatomic areas. ${ }^{9,17}$

Zauladek et al. reported that globular nevi were significantly more frequent in the upper trunk than in the middle trunk in patients aged from two to 20 years. ${ }^{12}$ That study excluded lesions on the head, neck, face, extremities, genitals and mucous membranes.

In our study, it was interesting to observe that, on the face, the prevalence of globules and network was inverted, where we saw a higher prevalence of globules than of network. A limitation to be considered is that, once we include lesions that are very small or new, the differentiation between dots and globules can be difficult to be made.

As presented, our observations were partially comparable with the findings of previous studies, but there were limitations.

Among the evaluated lesions, $37.3 \%$ presented a diameter smaller than $2 \mathrm{~mm}$. It is important to consider that lesions that are very new may not present defined patterns. These lesions may be able to evolve into more defined patterns in the future, which would change the proportion of the observed patterns when the nevus becomes bigger.

The aim of this work was to evaluate the dermoscopic structures and patterns of nevi, according to the location of the lesions.

Standardized studies, with larger sample size, as well as long-term follow-up of the lesions, can provide important answers related to the evolution and development of nevi, and shed further light on the mechanisms of nevogenesis.

\section{CONCLUSIONS}

In our study, the reticular pattern was the most commonly observed (39\%).

According to the body site, the reticular pattern was the most prevalent in the extremities.

In the study of the dermoscopic structures present in the lesions according to the studied area, we observed, on the face, an inversion in the prevalence of globules and network, with a higher prevalence of globules over the pigment network.] 


\section{REFERENCES}

1. Haliasos EC, Kerner M, Jaimes N, Zalaudek I, Malvehy J, Hofmann-Wellenhof $\mathrm{R}$, et al. Dermoscopy for the pediatric dermatologist part III: dermoscopy of melanocytic lesions. Pediatr Dermatol. 2013;30:281-93.

2. Cohen $B$. To biopsy or not to biopsy changing moles in children and adolescents: are we removing too many pigmented nevi in this age group? Comment on "Variables predicting change in benign melanocytic nevi undergoing short-term dermoscopic imaging" Arch Dermatol. 2011;147:659-60.

3. Scope A, Marghoob AA, Dusza SW, Satagopan JM, Agero AL, Benvenuto-Andrade $C$, et al. Dermoscopic patterns of naevi in fifth grade children of the Framingham school system. Br J Dermatol. 2008;158:1041-9.

4. Tcheung WJ, Bellet JS, Prose NS, Cyr DD, Nelson KC. Clinical and dermatoscopic features of 88 scalp naevi in 39 children. Br J Dermatol. 2011;165:137-43.

5. Hofmann-Wellenhof R. Special criteria for special locations 2: scalp, mucosal, and milk line. Dermatol Clin. 2013;31:625-36, ix.

6. Stolz W, Braun-Falco O, Bilek P, Landthaler M, Cognetta A. Color Atlas of Dermatoscopy. Oxford: Blackwell Science; 1994.

7. Aguilera P, Puig S, Guilabert A, Julià M, Romero D, Vicente $\mathrm{A}$, et al. Prevalence study of nevi in children from Barcelona. Dermoscopy, Constitutional and environmental factors. Dermatology. 2009;218:203-14.

8. Scope A, Marghoob AA, Chen CS, Lieb JA, Weinstock MA, Halpern AC, et al. Dermoscopic patterns and subclinical melanocytic nests in normal-appearing skin. Br J Dermatol. 2009;160:1318-21.

9. Krengel S. Nevogenesis-new thoughts regarding a classical problem. Am J Dermatopathol. 2005;27:456-65.

10. Unna, PG. The Histopathology of the Diseases of the Skin. New York: Macmillan \& Co.; 1896

11. Maize JC, Foster G. Age-related changes in melanocytic naevi. Clin Exp Dermatol. 1979;4:49-58

12. Zalaudek I, Schmid K, Marghoob AA, Scope A, Manzo M, Moscarella E, et al. Frequency of dermoscopic nevus subtypes by age and body site: a crosssectional study. Arch Dermatol. 2011;147:663-70.

13. Zalaudek I, Sgambato A, Ferrara G, Argenziano G. Diagnosis and management of melanocytic skin lesions in the pediatric praxis. A review of the literature. Minerva Pediatr. 2008:60:291-312

14. Zalaudek I, Grinschgl S, Argenziano G, Marghoob AA, Blum A, Richtig E, et al. Age-related prevalence of dermoscopy patterns in acquired melanocytic naevi. $\mathrm{Br}$ J Dermatol. 2006;154:299-304.

15. Oliveria SA, Geller AC, Dusza SW, Marghoob AA, Sachs D, Weinstock MA, et al. The Framingham school nevus study: a pilot study. Arch Dermatol 2004;140:54551.

16. Belloni Fortina A, Zattra E, Romano I, Bernardini B, Alaibac M. Clinical and dermoscopic features of nevi in preschool children in Padua. Dermatology. 2010;220:53.

17. Nordlund JJ. The lives of pigment cells. Dermatol Clin. 1986;4:407-18.
MAILING ADDRESS:

Christiane Donato Piazza

Rua Borges Lagoa 508

04038-001 - São Paulo - SP

Brazil

Email: christiane.piazza@gmail.com

How to cite this article: Piazza CD, Yamada S, Marcassi AP, Maciel MG, Seize MP, Cestari SCP. Dermoscopic patterns of melanocytic nevi in children and adolescents: a cross-sectional study. An Bras Dermatol. 2017;92(3):340-4. 